\title{
Clima organizacional y calidad de servicio of recido por la Universidad Nacional de Pilar
}

\author{
Alicia Fernández Cristaldo \\ alifer85@gmail.com
}

\section{RESUMEN}

La investigación tiene como objetivo determinar la manera que incide el clima organizacional en la calidad de servicios ofrecidos a la comunidad por la Universidad Nacional de Pilar. Se definen brevemente el clima organizacional contextualizando su estrecha relación con la calidad de servicios ofrecidos por las intuiciones como fenómeno vinculado a actitudes y la relación de éstas con el comportamiento y los resultados que hacen sea un tema de gran importancia en la actualidad reconocido por cada vez más como una necesidad. Permite examinar la institución educativa la que se ha convertido en una competencia muy fuerte para el mercado actual. Se ha llegado a la conclusión que existe un porcentaje elevado de la calidad de servicios ofrecidos, el grado de compromiso de los funcionarios y compromiso organización, no obstante, se observan porcentajes que requieren ser mejorados.

Palabras clave: Clima Organizacional; Calidad de Servicios; Funcionarios; Institución Educativa. 


\title{
Organizational climate and quality of service offered by the national university of Pilar
}

\begin{abstract}
The objective of the research is to determine how the organizational climate affects the quality of services offered to the community by the National University of Pilar. The organizational climate is briefly defined contextualizing its close relationship with the quality of services offered by intuitions as a phenomenon linked to attitudes and the relationship of content with behavior and results that make it a subject of great importance today recognized by each time more like a necessity. Lets examine the educational institution which has become a very strong competition for today's market. It has been concluded that there is a high percentage of the quality of services offered, the degree of commitment of officials and the organization of the commitment, however, there are percentages that need to be improved.
\end{abstract}

Keywords: Organizational Climate; Quality of Services; Officials; Educational institution.

Artículo recibido: 03 nov. 2020 Aceptado para publicación: 07 dic. 2020

Correspondencia alifer85@gmail.com Conflictos de Interés: Ninguna que declarar 


\section{INTRODUCCIÓN}

Las personas en las organizaciones son medios para lograr fines, por lo que se requiere dinamismos, creatividad y participación activa de sus integrantes, siendo que los trabajadores pueden decidir su eficacia, eficiencia y competitividad de una manera trascendental y sea reconocida y ocupe un estándar social determinado, tema en que se centra el presente artículo de revisión bibliográfica cuyo objetivo es describir la manera que incide el clima organizacional en la calidad de servicios educativos de funcionarios. Para tal efecto se definen brevemente el clima organizacional contextualizando su estrecha relación con la calidad de servicios ofrecidos en las intuiciones como fenómeno vinculado a actitudes y la relación de éstas con el comportamiento y los resultados que hacen sea un tema de gran importancia en la actualidad reconocido por cada vez más como una necesidad siendo que existen diferentes instituciones educativas que ofrecen servicios y todas ellas prometen calidad.

\section{EL PROBLEMA}

El clima organizacional hace propiamente referencia a la percepción del trabajador en lo que respecta a su lugar de trabajo, la toma de decisiones, las relaciones interpersonales entre los elementos de la organización, ya sea en los diferentes niveles jerárquicos y se formal e informal y el resultado de este ante tercero quienes son los clientes.

Una de las dificultades de la institución educativa es la gran competencia del mercado actual, presentado servicio similares por lo que se requiere reflejan los valores reales de cada institución, la calidad y por sobre todo el servicio que brinda a su cliente; por lo tanto, el clima organizacional tiene a ser ignorado por la mayoría de los responsables de la institución ya que dan por sentado que se proporcionan las herramientas básicas a sus empleados para desarrollar su trabajo y esto será suficiente para que se desempeñen eficientemente. Entendida la importancia y el impacto de la educación, los servicios que se brinden deben tener altos estándares de calidad, por lo que se hace necesario una adecuada medición de los mismos.

En la actualidad todas las empresas e instituciones que ofrecen servicios requieren aunar todo esfuerzo que les permita mantenerse y ganar buena imagen, y para ello, se requiere ofrecer servicio de calidad, demostrada en la responsabilidad de los empleados, reflejada en la actitud y comportamiento con la institución y los clientes que son los alumnos de las diferentes facultades, permitiendo la accesibilidad a los servicios, ofrecidos por la 
institución, manteniendo una buena convivencia entre compañeros de trabajo como también reflejada en la apariencia personal de los empleados.

Contar con funcionarios comprometidos con la organización, capaz de proveer las personas correctas en los momentos y lugares correctos para poder satisfacer tanto las necesidades individuales del personal, (seguridad en el empleo, reconocimiento, buen ambiente de trabajo, incentivos, salario justo, oportunidad de ascenso), como las organizacionales, el de ofrecer servicio de calidad al cliente (alumnos).

\section{OBJETIVOS}

\section{General}

Analizar la manera que incide el clima organizacional en la calidad de servicios ofrecidos a la comunidad por la Universidad Nacional de Pilar.

\section{Objetivos específicos}

1. Determinar la relación existente entre clima organizacional y calidad de servicios ofrecidos a la comunidad por los funcionarios de la Universidad investigada.

2. Establecer el grado de compromiso de los funcionarios con la institución a la que pertenecen.

3. Identificar la correspondencia entre clima organizacional existente y las expectativas de los funcionarios.

\section{JUSTIFICACIÓN}

Investigar el clima organizacional de la institución educativa permite estudiar la percepción de la comunidad quienes reciben servicios y poder medir la calidad de servicios ofrecidos, el grado de compromiso de los funcionarios y el grado de compromiso organizacional.

Una de las dificultades de la institución educativa es la gran competencia del mercado actual, presentado servicio similares por lo que se requiere reflejan los valores reales de cada institución, la calidad y por sobre todo el servicio que brinda a su cliente; por lo tanto, el clima organizacional tiene a ser ignorado por la mayoría de los responsables de la institución ya que dan por sentado que se proporcionan las herramientas básicas a sus empleados para desarrollar su trabajo y esto será suficiente para que se desempeñen eficientemente.

Con la investigación permitió examinar la institución educativa superior la que se ha convertido en una competencia muy fuerte para el mercado, y lo que merece una especial 
atención, viendo la necesidad del estudio del clima organizacional y determinar la calidad del servicio ofrecido a la comunidad.

\section{Método}

Se trata de una revisión descriptiva para cuya localización de documentos bibliográficos fueron utilizadas varias fuentes documentales de manera a describir diferentes aspectos de la realidad a analizar, en este caso el clima organizacional y calidad de servicios educativos de funcionarios de la Universidad Nacional de Pilar

El método mixto de investigación es el elegido para este trabajo; cualitativo y cuantitativo, para abarcar diferentes aspectos de la realidad a analizar, representado así representan el más alto grado de integración o combinación entre los enfoques cualitativo y cuantitativo agregando complejidad al diseño de estudio (Hernández, Fernández y Baptista, 2003).

La población estuvo constituida por las siete Facultades de la Universidad Nacional de Pilar, las que se citana continuación: Facultad de Humanidades y Ciencias de la Educación, Facultad de Ciencias Aplicadas, Facultad de Derechos, Ciencias Políticas y Sociales, Facultad de Ciencias Contables, Administrativas y Económicas, Facultad de Ciencias Agropecuarias y Desarrollo Rural, Facultad de Ciencias, Tecnologías y Artes y la Facultad de Ciencias Biomédicas, de los cuales se tomó el 30\% de alumnos de cada facultad de manea aleatoria.

\section{REVISIÓN BIBLIOGRÁFICA}

\section{Clima Organizacional y Calidad de Servicios}

\section{Las Organizaciones y Clima organizacional}

De acuerdo a Sotelo Asef y Figueroa González, (2017):

En la actualidad las organizaciones se encuentran en una etapa de competencias, debido a lo cual tienen que estar en óptimas condiciones desde su interior para brindar un buen servicio, de ahí que los conceptos de clima organizacional y calidad en el servicio sean fundamentales para fortalecer su razón de ser y por consecuencia poder tomar decisiones que las lleven a desarrollarse dentro del contexto de la competitividad y sus directivos coadyuven a la mejora de las instituciones.

De acuerdo a Gronroos (1994), mencionado por Lascurain, (2012), considera que existen tres dimensiones de la calidad en el servicio que son: 
- Calidad técnica: Involucra lo que el cliente está recibiendo del proveedor. Esto puede ser medido por parte del cliente de manera más objetiva.

- Calidad funcional: Involucra la manera en que el servicio es entregado. Esto se refiere a la interacción psicológica entre el comprador y el proveedor. Es percibida en una manera bastante subjetiva y pueden influir elementos como: actitud y comportamiento de los empleados, acercamiento del personal de servicio, accesibilidad al servicio, apariencia del personal, relación entre los empleados y relación entre los empleados y el cliente.

- Imagen corporativa: Es la dimensión de la calidad que resulta de cómo los clientes perciben a la empresa y se espera que sea construida principalmente por la dimensión técnica de la calidad ya que afecta la percepción del servicio por parte del cliente (p.3). Para la presente investigación se está estudiando específicamente la calidad funcional, es decir, la que entrega servicio educativo a la comunidad.

Es sabido que el clima organizacional experimentado por el empleado está determinado por el reto laboral que plantea el puesto, la claridad del trabajo, la supervisión y los incentivos, todos estos son factores organizacionales que intervienen en la calidad del servicio ofrecido por una institución.

De acuerdo a Chiavenato (2011). La organización es definida "como una entidad o unidad coordinada que le permite a la sociedad perseguir logros que no se pueden obtener por individuos actuando solos" (p. 78).

"El clima organizacional debe considerarse como intersubjetivo, es decir, como una vivencia diaria entre los colaboradores de una institución educativa donde intercambian percepciones” (Edel, García y Guzmán, 2007).

"El clima Organizacional, es un conjunto de percepciones que los individuos tienen de una empresa y el entorno, independientemente de cómo lo perciben otros, por lo tanto, es más una dimensión del individuo que de la organización” (Cota Luevano, 2017).

El estudio de las organizaciones data de varios años, estudiosos como Fayol, Taylor, Chiavenato, se han centrado en el comportamiento de las organizaciones y la dinámica de éxitos de los mismos, afectando la productividad de la misma, determinando el clima laboral en las empresas y su estrecha relación con el nivel de satisfacción que concibe el empleador dentro de la organización, un aspecto reflexionada en el mundo empresarial y organización, especificándose la motivación constante como resultado, de empleados más comprometidos a nivel institucional. 
El clima laboral, se compone de un gran número de factores, tanto físicos como emocionales, que inciden en el comportamiento y desempeño de los integrantes del equipo de trabajo (García, 2009). El clima Organizacional determina la forma en que un individuo percibe su trabajo, su desempeño, productividad y satisfacción, contemplando el medio ambiente humano y físico en el que se desarrolla el trabajo cotidiano, influyendo así en la satisfacción del personal y por lo tanto en la productividad, y es sabido que las grandes variaciones significativas en la productividad de la empresa, pueden puede estar directamente relacionado con el clima laboral de la organización.

Las organizaciones desean ser más competitivas y deben preocuparse en mejorar sus procesos llevándolos a cabo con personal debidamente preparado para alcanzar los resultados esperados y sobresalir ante la competencia; es por ello que en la actualidad las empresas que quieran plantear de forma acertada su futuro deben estructurarse con una misión clara y directa hacia sus empleados y clientes. La organización invierte recursos con cada colaborador al seleccionarlo, incorporarlo y capacitarlo. Para proteger esta inversión, la organización debería conocer el potencial de sus hombres. Esto permite saber si cada persona ha llegado a su techo laboral o puede alcanzar posiciones más elevadas. También permite ver si hay otras tareas de nivel similar que puede realizar, desarrollando sus aptitudes y mejorando el desempeño de la empresa (Cota Luevano, 2017, p 14).

Identificar los elementos que intervienen en el clima organizacional, puede destacar las circunstancias en las que un empleado se desenvuelve en sus actividades diarias y cómo el resultado de éstas si son inadecuado, puede generar como resultado insatisfacción en los trabajadores que prestan sus servicios dentro de las organizaciones como así también si son adecuadas genera productividad.

De acuerdo a estudios, existen organizaciones preocupados y ocupados por invertir y conseguir un ambiente adecuado para sus trabajadores, con la finalidad que, estos generen resultados favorables para la institución, centra su problemática en la relación directa entre el clima organizacional y el rendimiento productivo.

\section{Servicios Educativos y Compromiso organizacional}

Según Cota Luevano, (2017):

La educación es un proceso social que tiene por encargo la formación integral del ser humano para la vida. Ello exige a los sistemas educativos que enfrenten eficazmente los 
procesos formativos e incidan en los problemas sociales como la multicultura, la tecnología, las necesidades de convivencia, las sociedades multiétnicas, la universalización de los esfuerzos en contra al cambio climático, la conservación del planeta y el ser humano (p.12).

En la presente revisión se está exponiendo la intervención el clima laboral de cualquier organización, la percepción que tengan los trabajadores del ambiente donde se encuentran trabajando y en la que intervienen la estabilidad del trabajo, el trabajo en equipo, la buena comunicación, por lo tanto, el resultado de este ante tercero que son en este caso los estudiantes.

De acuerdo a Pilligua y Arteaga Ureta, (2019). El clima Organizacional determina la forma en que un individuo percibe su trabajo, su desempeño, productividad y satisfacción, contemplando el medio ambiente humano y físico en el que se desarrolla el trabajo cotidiano, influyendo así en la satisfacción del personal y por lo tanto en la productividad. La noción de rendimiento - productividad, se encuentra vinculada a la proporción existente, entre los recursos que se emplean para conseguir algo y el resultado que luego se obtiene. De este modo, el rendimiento se asocia al beneficio o la utilidad (Pérez \& Merino, 2016).

Pérez \& Gardey (2012), sostienen que: "la mejor productividad supone una mayor rentabilidad en cada empresa". Con relación a lo anterior, la productividad puede definirse como una medida económica que, calcula cuántos bienes y servicios se han producido por cada factor utilizado (trabajador, capital, tiempo, costes, etc.) durante un periodo determinado (Sevilla, 2015). Mide la eficiencia de producción por cada factor o recurso utilizado, entendiendo por eficiencia, el hecho de obtener el mejor o máximo rendimiento, utilizando un mínimo de recursos.

\section{Calidad de Servicio}

Salazar Yépez y Cabrera Vallejo (2016):

Debido al nacimiento de este mercado universitario y a las demandas de la nueva economía global, los gestores en el sector universitario están buscando nuevos caminos de dirigir y mejorar la calidad de la enseñanza superior. Para ello, deben conocer la valoración de la calidad de servicio que se presta y sumergirse en un proceso de mejora continua que les permita estar siempre abiertos a nuevas maneras de trabajar, más adecuadas y acordes con los tiempos (p.20). 
Salazar Yépez y Cabrera Vallejo (2016) consideran que:

Actualmente, la importancia de ofrecer servicios académicos de calidad es cada vez más esencial en el mundo. Los usuarios son más exigentes y tienen una gran noción de lo que implica la calidad. Para cumplir con estas expectativas, se han desarrollado numerosas técnicas y herramientas para medir y asegurar la calidad de los productos ofrecidos por el sector. Sin embargo, en el caso de los servicios, esto es aún más complejo, dada la naturaleza subjetiva que los caracteriza. Según el contexto, la calidad de los servicios educativos es esencial para el desarrollo de un país. Además, se ha demostrado amplia y sobradamente que la mejora de la calidad de la educación contribuye al alcance de toda una serie de objetivos en el plano del desarrollo económico y social. Las organizaciones que se dedican a la educación y formación, tanto pública como privada dan un servicio a la sociedad. Las personas, independientemente de la edad, concurren a ellas para adquirir una serie de competencias que les capaciten para actuar e integrarse en la sociedad (p.23). La globalización de la economía, la tendencia hacia el incremento de la competencia por parte de las organizaciones y la búsqueda de la excelencia organizativa, no han dejado al margen a la formación y más en concreto a la educación ofrecida en las instituciones educativas.

El clima organizacional puede ser un vínculo u obstáculo para el buen servicio de la institución, puede ser factor de distinción e influencia en el comportamiento de los recursos humanos que los integra ser parte de instrumentos útiles para la organización, orientando sus esfuerzos hacia los aspectos que están contribuyendo a afectar positiva en la productividad y proveer servicios de calidad.

\section{Compromiso organizacional de funcionarios}

Hernández Hernández y Castro Cortez (2015):

En pleno siglo veintiuno cobra vigencia cada vez más la premisa acerca de que las organizaciones para tener ventajas competitivas y ser sostenibles en el tiempo, deben caracterizarse por el respeto como eje transversal en su accionar diario con sus interlocutores y muy especialmente con sus trabajadores, logrando establecer vínculos o lazos entre estos y la organización, incrementando así su compromiso y productividad. Se trata de establecer una simbiosis entre empleador y empleado, proporcionándole lo que necesita, y éste recíprocamente deberá aportar todo el valor posible a la empresa. García, Carreón, Hernández y Morales (2014): 
El compromiso laboral está imbricado en un sistema psicológico organizacional que parte del clima y termina en la satisfacción. En tal sistema, el compromiso interrelaciona con el liderazgo y el desempeño para mediar la relación entre clima y satisfacción. Se trata de supuestos según un incremento en las relaciones de tareas y humanas impacta el liderazgo, compromiso y desempeño para incidir sobre un aumento en la satisfacción de vida.

\section{Hernández Hernández y Castro Cortez (2015):}

A partir de las referencias mencionadas, se puede decir que el compromiso organizacional consiste en el fuerte vínculo intrínseco de identificación e involucramiento de una persona con una determinada organización, generando conductas externas (la consecuencia del compromiso) de apropiación y defensa que en definitiva son beneficiosas para la organización por ser hechos palpables y medibles. Una especie de apego psicológico que siente el individuo por la organización, asimilando características de ésta como propias y actuando en pro y beneficio de ambos. En función de ello se puede decir que, la base del compromiso organizacional es en definitiva el sentido de pertenencia que siente un trabajador hacia la empresa, dándose una conexión que le hace sentirse como parte de ella.

Sobre la base de lo antes expuesto, Gracia (1999) refiere que "el compromiso organizacional consiste en la intensidad de la participación de un empleado y su identidad con la organización”. (p.11). Compromiso éste que se caracteriza por: creencia y aceptación de las metas y los valores de la organización. Disposición a realizar un esfuerzo importante en beneficio de la organización. Deseo de mantenerse dentro de la organización. Por ello, el compromiso organizacional es una actitud más amplia que la satisfacción, porque se aplica a toda la organización y no sólo al trabajo.

En tanto para Meyer, Stanley, Herscovitch, y Topolnytsky, (2002). mencionado por Hernández Hernández y Castro Cortez (2015) se distinguen tres formas de compromiso organizacional: a) compromiso afectivo, donde el individuo fuertemente comprometido se identifica con, está involucrado en y disfruta ser miembro de la organización; b) compromiso de continuación, que expresa el costo percibido asociado con el abandono de la organización; y c) el compromiso normativo, que refleja una obligación percibida de permanecer en la organización (p.6). 
En relación con lo antes dicho y dada la importancia evidente que para la empresa supone el contar con empleados comprometidos; se considera que para lograrlo es necesario desarrollar la confianza en cada uno de los trabajadores generando como consecuencia, el comportamiento voluntario positivo y actitud proactiva de cada uno de ellos, forjando beneficios no solo para la empresa como lo pudiera ser la alta productividad, sino que también propiciara un clima laboral óptimo para él y sus compañeros de trabajo (Hernández Hernández y Castro Cortez, 2015).

Según Hernández, Barrios y Martínez (2018).

La gestión de calidad resulta hoy día una estrategia para impulsar la competitividad empresarial que permite, desde una perspectiva integral, observar la organización como un conjunto de procesos interrelacionados cuyo fin último es, entre otros, lograr la satisfacción del cliente.

Sin duda, el servicio al cliente constituye para las organizaciones una de las grandes exigencias que demanda adecuada atención para lograr una impecable prestación del mismo. Debido a su relevancia, las empresas realizan un gran esfuerzo por entregar a sus clientes este conjunto de actividades que implican una naturaleza propia, con el fin de lograr que el usuario se sienta complacido y lo encuentre acorde con el valor invertido por su adquisición. Sin embargo, existen factores que influyen en la entrega del servicio al cliente, los cuales deben ser revisados para establecer cómo intervenirlos, con el fin de mejorar esas acciones.

Cuando los elementos: Identificación, membresía y lealtad están presentes en el trabajador -capital humano-, se puede decir que la organización tiene personas comprometidas; es decir, personas que buscan cumplir sus metas desempeñándose satisfactoriamente, que se sienten miembros activos de la empresa y que la defienden mostrando lo positivo de ella. Es claro que para generar el compromiso organizacional es necesario propiciarlo, no basta el conocimiento para resolver problemas, para mejorar la condición humana, es necesario cultivar la capacidad emocional del activo más importante de las empresas el trabajador (Drucker 2002); para lograr más fácil y rápidamente las metas organizacionales, en beneficio de todos. De esta manera es idóneo para las investigadoras señalar que la responsabilidad social acarrea un nivel de compromiso sumamente elevado para las organizaciones, porque es llevar a otro nivel la 
razón de ser de la empresa considerando a todas las personas que puedan ser afectadas, cobrando vigencia la Teoría de las partes interesadas.

Brindar un ambiente laboral propicio es un tema de suma importancia para cualquier organización. El talento humano es la fuerza que mueve cualquier proceso, facilita el desarrollo del mismo y, sin duda alguna, fortalece el progreso continuo tanto de los empleados como de la organización. Es relevante no solo suplir las necesidades básicas del empleado; de por medio aparece una gran variedad de necesidades personales que influyen directamente en los comportamientos y actitudes que el trabajador tiene dentro de la organización; por esto dichas necesidades deben ser ajustadas dentro de la empresa para que la calidad de vida laboral del empleado sea óptima (Nair, 2013, p.18)

García Rubiano y Forero Aponte (2019).

$\mathrm{Al}$ respecto, consideran que la literatura tiene una serie de debates sobre la forma en que se debe llevar a cabo el cambio organizacional, pero siempre coinciden en la idea de que se debe instaurar enfocado en que los miembros de la organización reconozcan la necesidad de dicho proceso.

La investigación concluyó que la calidad de vida en el trabajo requiere cambios: en la forma de ver y de hacer las labores, en la manera de llevarlas a cabo; en la manera de conducir la organización; en las variantes de participación de las personas, y en el contexto de las responsabilidades, entre otros. Esto no significa que sea necesario cambiarlo todo, sino que el progreso en materia de calidad demanda adecuaciones en diferentes aspectos de la administración que constituyen un obstáculo para su logro. Esto conlleva la necesidad de promover acciones que impulsen el desarrollo local sostenible, entendido como una responsabilidad compartida por todos y cada uno de los agentes sociales, económicos y medioambientales, los cuales, de una forma u otra, tienen algo que proponer y recomendar para mejorar continuamente la calidad de vida de los trabajadores

De esta manera, la capacidad de cambio implica un enfoque sistémico para el desarrollo de la organización, de manera a aprovechar la capacidad natural de cada persona integrante de la organización, sus percepciones respecto a la equidad en relación inserción laboral, movilidad laboral o escalafones, salario, cumplimiento de reglamento organizacional, designación de tareas y los equipos de trabajo que deseen unirse al cambio 
y colaborar con la organización para ubicarlo en lo más altos niveles a nivel organizacional.

\subsection{Satisfacción laboral}

Según Ruiz Segura (2009).

La Satisfacción Laboral es una de las principales actitudes frente al trabajo, y un predictor del comportamiento tanto en el ámbito laboral como personal, por lo tanto, su medición puede ser considerada como una herramienta útil para diagnosticar la salud de la empresa e identificar problemas latentes que afecten tanto a los trabajadores como a la organización.

La Satisfacción Laboral en la actualidad es un tema de gran interés, relevante para las organizaciones, estudia como una variable que influye sobre las interrelaciones entre individuos y la organización, pues de esta interacción es que resulta necesario prestar atención a ambos intereses. En otras palabras, las personas necesitan a las organizaciones como importantes medios para alcanzar sus metas y a su vez estas entidades necesitan a las personas para lograr sus objetivos organizacionales (Ruiz Segura, 2009).

$\mathrm{Al}$ respecto, es importante considerar que actualmente los estudiantes buscan la mejor opción en el mercado educativo, por lo que representa la importancia que tienen los estudiantes como consumidores y quienes requieren recibir calidad de servicio, por lo tanto como responsables de toda organización y en caso necesario y la que siempre se presenta, se deben tomar correctivos para garantizar la calidad de servicio ofrecido en todos sus aspectos de tal manera posicionarse dentro de un mercado tan competitivo como son actualmente las universidades.

Según Barrientos (2017). Hay tres campos que son de interés para la organización si considera que su empresa debe ser competitiva, esto es, lo que significa el esfuerzo que hace la empresa por ser distinta de sus competidores directos e industrias sustitutas. La competitividad lo demuestra: cuando la empresa tiene la posibilidad de enfrentar sus productos con los otros proveedores y sale ganadora, obtiene la preferencia de sus clientes.

Según Wellington, (1997), mencionado por Salazar Yépez y Cabrera Vallejo (2016) sostiene que el servicio al cliente "son aquellos elementos que se debe entregar de acuerdo a las necesidades del cliente". El cliente es aquella persona u organización que adquiere o compra, es el motivo principal por el que se crea, produce, fabrica y comercializa 
productos y servicios, por lo que la competencia por parte de las empresas y la búsqueda de la excelencia organizativa, no han dejado al margen a la formación y más en concreto a la educación ofrecida en las universidades y las que requieren ser atenidas como prioridad organizativa si se quiere permanecer en el mercado y sobre todo establecer nuevas metas de expansión de nuevos mercados.

\section{ANÁLISIS Y DISCUSIÓN}

Figura: Calidad de servicios ofrecidos-Grado de compromiso de los funcionarios y

Compromiso organizacional

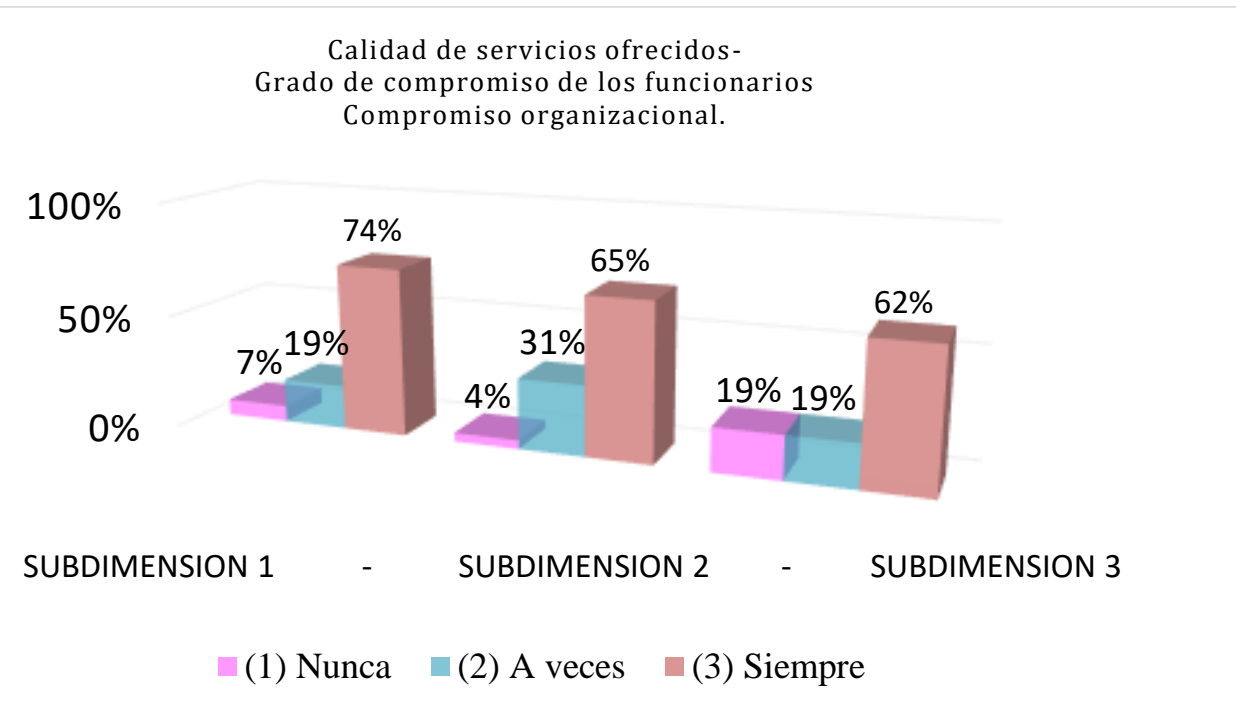

Los resultados revelan porcentajes elevados de la calidad de servicios ofrecidos, el grado de compromiso de los funcionarios y compromiso organización, no obstante, se observan porcentajes que requieren ser mejorados.

De acuerdo a Bernardez (2006) considera "una organización es una colectividad con una frontera parcialmente identificable, un orden normativo, niveles de autoridad, sistemas de comunicaciones y sistemas de coordinación de membresías; esta colectividad existe de manera continua en un ambiente y se involucra en actividades que se relacionan por lo general con un conjunto de metas; las actividades tienen resultados para los miembros de la organización, la organización misma y la sociedad" (p. 54).

En la actualidad todas las empresas e instituciones que ofrecen servicios requieren aunar todo esfuerzo que les permita mantenerse y ganar buena imagen, y para ello, se requiere ofrecer servicio de calidad, demostrada en la responsabilidad de los empleados, reflejada en la actitud y comportamiento con la institución y los clientes que son los alumnos de las diferentes facultades, permitiendo la accesibilidad a los servicios, ofrecidos por la 
institución, manteniendo una buena convivencia entre compañeros de trabajo como también reflejada en la apariencia personal de los empleados.

Contar con funcionarios comprometidos con la organización, capaz de proveer las personas correctas en los momentos y lugares correctos para poder satisfacer tanto las necesidades individuales del personal, (seguridad en el empleo, reconocimiento, buen ambiente de trabajo, incentivos, salario justo, oportunidad de ascenso), como las organizacionales, el de ofrecer servicio de calidad al cliente (alumnos).

\section{CONSIDERACIONES FINALES}

Investigar el clima organizacional de la institución educativa permite estudiar la percepción de la comunidad quienes reciben servicios y poder medir la calidad de servicios ofrecidos, el grado de compromiso de los funcionarios y el grado de compromiso organizacional.

El clima organizacional hace propiamente referencia a la percepción del trabajador en lo que respecta a su lugar de trabajo, la toma de decisiones, las relaciones interpersonales entre los elementos de la organización, ya sea en los diferentes niveles jerárquicos y se formal e informal y el resultado de este ante tercero quienes son los clientes.

Una de las dificultades de la institución educativa es la gran competencia del mercado actual, presentado servicio similares por lo que se requiere reflejan los valores reales de cada institución, la calidad y por sobre todo el servicio que brinda a su cliente; por lo tanto, el clima organizacional tiene a ser ignorado por la mayoría de los responsables de la institución ya que dan por sentado que se proporcionan las herramientas básicas a sus empleados para desarrollar su trabajo y esto será suficiente para que se desempeñen eficientemente. Entendida la importancia y el impacto de la educación, los servicios que se brinden deben tener altos estándares de calidad, por lo que se hace necesario una adecuada medición de los mismos.

A partir de las perspectivas antes analizadas, en este artículo se comparte el criterio de los autores que consideran ambos constructos diferentes y relacionados. Diferentes porque el clima es descriptivo de la organización y la satisfacción es evaluativo del funcionario, en estrecha relación por desarrollarse en un contexto continuo, duradero y conectados como es el clima organizacional y su efecto a la satisfacción de los trabajadores y a su vez esta satisfacción incide en el clima organizacional y a la calidad de servicio ofrecido a los estudiantes. 
El clima organizacional es un referente de gran importancia en las organizaciones, que pretenden ser competitivas y que se preocupan por el desempeño de los funcionarios quienes trabajan en la organización y donde están en un proceso de continuo mejoramiento del ambiente de su organización, buscando incrementar los niveles de productividad, prestando mayor consideración al personal; donde el bienestar en las personas y la motivación que tengan, son los pilares básicos para ser productivos, basados en la eficiencia y eficacia sinónimo de productividad.

\section{REFERENCIA BIBLIOGRÁFICA}

Barrientos Felipa, P. (2017). Marketing + internet = e-commerce: oportunidades y desafíos. Revista Finanzas y Política Económica, vol. 9, núm. 1, enero-junio, 2017, pp. 41-56 Universidad Católica de Colombia Bogotá, Colombia

Bernardez, M. (2006). Desempeño Organizacional. Pensilvania, Estados Unidos de América: AuthorHouse.

Caykoylu, S., Egri, C., Havlovic, S. \& Bradley, C. (2011). Key organizational commitment antecedents for nurses, paramedical professionals and non-clinical staff. Journal of Health Organization and Management, 25(1), 7-33.

Chiavenato, I. (2011). Administración de Recursos Humanos. México: Mcgraw Hill.

Cota Luevano, J. A. (2017). Método para evaluar el clima organizacional del área docente de un centro de bachillerato tecnológico de cd. Obregón, sonora Ciencias Administrativas, núm. 10, Universidad Nacional de La Plata, Argentina

Cuesta, M. (2009). Introducción al muestreo. Universidad de Ovideo.

Cuesta, M. (2009). Introducción al muestreo. Universidad de Ovideo.

Edel R, García A, Guzmán F. (2007). Clima y compromiso organizacional. Disponible en http://eumed.net/libros/

Edel R, García A, Guzmán F. (2007). Clima y compromiso organizacional. Disponible en http://eumed.net/libros/

García Lirios, C; Carreón Guillén, J; Hernández Valdés, J; Morales Flores, ML (2014) Contraste de un modelo del compromiso laboral en centros de salud pública Acta Universitaria, vol. 24, núm. 1, enero-febrero, 2014, pp. 48-59 Universidad de Guanajuato Guanajuato, México

García Rubiano, M; Forero Aponte, C. (2019). Calidad de Vida Laboral y la Disposición al Cambio Organizacional en Funcionarios de Empresas de la Ciudad de Bogotá 
- Colombia Acta Colombiana de Psicología, vol. 19, núm. 1, 2016, pp. 79-90 Universidad Católica de Colombia Bogotá, Colombia

Gracia, M. (1999). Autoestima y compromiso organizacional. Revista Investigación Psicológica Vol. --- $\mathrm{N}^{\circ} 10$

Hernández Hernández, G.; Castro Cortez, D (2015). Responsabilidad social como estrategia activadora del compromiso organizacional de los trabajadores Negotium, vol. 10, núm. 30, abril, 2015, pp. 123-141 Fundación Miguel Unamuno y Jugo Maracaibo, Venezuela

Hernández Palma H, Barrios Parejo, I, Martínez Sierra D (2018). Gestión de la calidad: elementos clave para el desarrollo de las organizaciones (en línea) https://doi.org/10.18041/1900-0642/criteriolibre.2018v16n28.213

Hernández, R., Fernández, C. y Baptista, P. (2003). Metodología de la investigación (3 ed.). México: Editorial Mc Graw-Hill

Hernández, R., Fernández, C. y Baptista, P. (2003). Metodología de la investigación (3 ${ }^{\mathrm{a}}$ ed.). México: Editorial Mc Graw-Hill

Lascurain, I. (2012). Diagnóstico y propuesta de mejora de la calidad en el servicio de una empresa de unidades de energía eléctrica interrumpida. México D.F: Tesis

Lascurain, I. (2012). Diagnóstico y propuesta de mejora de la calidad en el servicio de una empresa de unidades de energía eléctrica interrumpida. México D.F: Tesis

Pérez, J., \& Gardey, A. (2012). Definición de rendimiento. Obtenido de sitio web de: http://definicion.de/rendimiento/

Pilligua Lucas, C. F.; Arteaga Ureta., F. M. (2019). El clima laboral como factor clave en el rendimiento productivo de las empresas. estudio caso: Hardepex Cía. Ltda. Cuadernos Latinoamericanos de Administración, vol. XV, núm. 28, 2019 Universidad El Bosque, Colombia Disponible en: http://www.redalyc.org/articulo.oa?id=409659500007

Pupo-Guisado, B; Velázquez-Zaldívar, R; Tamayo-Fajardo, M. (2017). Relación entre clima organizacional y satisfacción laboral en empresas holguineras Ciencias Holguín, vol. 23, núm. 4, octubre-diciembre, 2017, pp. 1-14 Centro de Información y Gestión Tecnológica de Holguín Holguín, Cuba

Ruiz Segura, C, (2009). Nivel de satisfacción laboral en empresas públicas y Privadas de la Ciudad de Chillán. Memoria para optar al título de Contador Público y 
Auditor con Mención en Control de Gestión. Universidad del Bío-Bío. Facultad de Ciencias Empresariales Departamento de Gestión Empresarial.

Salazar Yépez, W; Cabrera-Vallejo, M (2016). Diagnóstico de la calidad de servicio, en la atención al cliente, en la Universidad Nacional de Chimborazo - Ecuador Industrial Data, vol. 19, núm. 2, julio-diciembre, 2016, pp. 13-20 Universidad Nacional Mayor de San Marcos Lima, Perú

Sevilla, A. (2015). Productividad. Obtenido de sitio web: http://economipedia.com/definiciones/productividad.html

Sotelo Asef, J. G, \& Figueroa González, E G. (2017). El clima organizacional y su correlación con la calidad en el servicio en una institución de educación de nivel medio superior. RIDE. Revista Iberoamericana para la Investigación y el Desarrollo Educativo, 8(15), 582-609. https://dx.doi.org/10.23913/ride.v8i15.312

Sotelo Asef, J. G, \& Figueroa González, E G. (2017). El clima organizacional y su correlación con la calidad en el servicio en una institución de educación de nivel medio superior. RIDE. Revista Iberoamericana para la Investigación y el Desarrollo Educativo, 8(15), 582-609. https://dx.doi.org/10.23913/ride.v8i15.312 